\title{
Advancing Scalability and Impacts of a Teacher Training Program for Promoting Child Mental Health in Ugandan Primary Schools: Protocol for a Hybrid- Type II Effectiveness-Implementation Cluster Randomized Trial
}

Keng-Yen Huang ( $\square$ huangk01@nyumc.org )

NYU School of Medicine https://orcid.org/0000-0003-3245-7614

Janet Nakigudde

Makerere University Faculty of Medicine: Makerere University College of Health Sciences

Elizabeth Nsamba Kisakye

Ministry of Education

Hafsa Sentongo

Republic of Uganda Ministry of Health

Tracy A Dennis-Tiwary

Hunter College CUNY: Hunter College

Yesim Tozan

New York University

Hyung Park

New York University Grossman School of Medicine

Laurie Miller Brotman

New York University Grossman School of Medicine

\section{Study protocol}

Keywords: School Program, Mental Health, Prevention, Effectiveness-Implementation, Uganda, SubSaharan-Africa, Scale-Up Strategy, Task Shifting, Hybrid-Type II, LMIC

Posted Date: May 25th, 2021

DOI: https://doi.org/10.21203/rs.3.rs-542401/v1

License: (c) (1) This work is licensed under a Creative Commons Attribution 4.0 International License.

Read Full License 


\section{Abstract}

Background: There are numerous challenges to promoting child mental health in low-and-middle income countries (LMICs). Most evidence-based interventions (EBIs) adapted to LMICs have not been scaled widely or evaluated for effectiveness and underlying mechanisms. Most mental health EBIs in LMICs rely on a task-shifting approach of implementation because of the shortage of mental health professionals; however, challenges related to task-shifting are understudied. Most EBIs have not been implemented using a scalable and sustainable model that leverages and strengthens existing structures. Through a cross-sector collaboration, we are carrying out a second generation investigation of implementation and effectiveness of a school-based mental health EBI for early childhood students that uses task-shifting and builds on existing educational structures. The ultimate shared goal of this collaboration is to scale and sustain the EBI country wide. The systems-level approach to promoting child mental health builds on ParentCorps, an EBI implemented in schools that has been shown in multiple trials to yield long-term benefits on mental health and school performance. Previous studies in Uganda found that ParentCorps Professional Development (PD) for teachers resulted in short-term benefits for classrooms, children and families.

Objectives: This paper describes the rationale and protocol for an effectiveness-implementation study of ParentCorps-PD for teachers in urban and rural Ugandan schools. The study considers the added value (i.e. impact and costs) of a brief wellness intervention for teachers in their task-shifting role and explores mechanisms and outcomes.

Methods: Using a hybrid-type II effectiveness-implementation cluster randomized controlled trial (cRCT), we will randomize 36 schools (18 urban and 18 rural) with 540 teachers and nearly 2,000 families to one of three conditions: $P D+$ Teacher-Wellness $(P D T), P D$ alone $(P D)$, and control. Primary effectiveness outcomes are teachers' use of mental health promoting strategies, teacher stress management, and child mental health. Mixed methods will be employed to examine underlying mechanisms of implementation and impact as well as cost-effectiveness.

Discussion: This research will generate important knowledge regarding the value of an EBI in urban and rural communities in a LMIC, and efforts toward supporting teachers who are task-shifting as a potentially cost-effective strategy for promoting child mental health.

Trial Registration: This trial was registered with ClinicalTrials.gov (registration number: NCT04383327; https://clinicaltrials.gov/ct2/show/NCT04383327) on May13, 2020.

\section{Contributions To The Literature}

- The study investigates the effectiveness and underlying effectiveness-implementation mechanisms of an EBI for promoting primary school students' mental health. Knowledge gains from this theoryguided EBI can be applied to scaling school-based mental health EBIs in other LMICs or low-resource communities. 
- The study investigates a task-shifting workforce implementation strategy (stress management) that has potential to improve the uptake, effectiveness, and sustainment of EBI practices.

- The study investigates transferability of an EBI developed from the US to urban and rural settings in a LMIC. Knowledge gained from this scale-up implementation research will inform future programming and policy decisions.

\section{Background}

Children under age 15 in Uganda comprise 47\% of the total population [1] and face enormous health and educational challenges [2]. More than one-quarter of Ugandan children have mental health problems [3], and only $53 \%$ achieve grade-level academic competency in 6th grade [4]. To address the mental health and educational burden in LMICs, providing population-level preventive services to promote child mental health has become a global priority. Despite emerging research suggesting the feasibility of adapting and transporting existing child mental health evidence-based interventions (EBIs) to Sub-Saharan Africa (SSA) [5-7], large-scale effectiveness research with diverse populations, and sustainable and scalable approaches in SSA are still lacking. In addition, most mental health EBIs in LMICs rely on a task-shifting approach to implementation because of the shortage of mental health professionals (MHPs); however, challenges related to task-shifting (e.g., workforce stress and job burnout) have rarely been addressed. For task-shifting to be successful, strategies to overcome challenges faced by the workforce and understanding mechanisms to support effective task-shifting are of paramount importance. We designed this research protocol to address implementation and effectiveness research gaps in LMICs. It investigates a mental health school-based EBI implemented by teachers in urban and rural Ugandan schools and examines the value of a brief intervention to support teachers in their task-shifting role. The protocol considers context and mechanisms of change to advance a scalable and sustainable EBI country-wide.

\section{Population-Level Approach to Child Mental Health Promotion in LMICs}

Young children in LMICs spend a considerable amount of time in school; In Uganda, 95\% of children are enrolled in primary schools ( 23\% enrollment in pre-primary schools) [8, 9]. Although the Ugandan child and adolescent mental health policy prioritizes the engagement of communities and child-serving institutions to contribute to mental health promotion efforts [10], school-based mental health interventions have not been widely applied in Uganda or in LMICs more generally. Population-level or universal school-based physical health programs have been found to be effective and cost-effective in addressing a wide range of individual, family, and service needs [11-13]. A school-based approach to mental health promotion has the potential to be feasible and a sustainable strategy to reach the majority of Ugandan children. Importantly it is aligned with national policy priorities for cost-efficient mental health promotion.

The EBI: ParentCorpsParentCorps is a multi-component school-based intervention that promotes early childhood mental health and development; it was built on an extensive body of cross-cultural parenting 
and child development research [14-21]. ParentCorps includes two components to support teachers and families to create environments that are safe, nurturing and predictable for children: 1) Professional Development (PD) for teachers on family engagement and social-emotional learning (SEL); 2) Family Program to support children's development of social-emotional competencies and self-regulation skills that are foundational for mental health. Together, the $P D$ and Family Program components encourage consistent use of a set of strategies by teachers and families. To achieve population-level reach and impact, ParentCorps is embedded in early childhood education programs as part of the normative school experience for all children. Two cluster randomized controlled trials (RCTs) in the United States (US) found that ParentCorps resulted in a broad range of long-term benefits for children from low-income families, including better mental health and academic performance three years post-intervention $[11,15$, $22,23]$.

In the US, ParentCorps MHPs provide PD to teachers and school-based MHPs. School-based MHPs implement the Family Program. Because most LMICs do not have school-based MHPs [24, 25], implementing $P D$ and the Family Program with existing resources is not feasible or scalable. Given the resource limitations and calls to provide preventive intervention more broadly in LMICs, we carried out a series of investigations to test one of the two components of ParentCorps $-P D$ only- in urban Uganda (RCT in 10 schools) [26] and rural Nepal (pre-post change in 30 schools)[27]. In both countries, we considered $P D$ to facilitate task shifting of mental health promotion from mental health professionals to classroom teachers $[28,29]$. The $P D$ (training and coaching) was intended to help teachers to create classroom environments that support SEL and mental health, to engage in mental health promoting interactions with students in the classroom and with parents during formal and informal interactions. The focus of $P D$ about universal strategies for all children and coaching extended application of these learnings to students and families with specific mental health needs.

Our previous $P D$ implementation in LMICs was carried out in cross-agency collaboration with the Ministry of Education (MOE) and Ministry of Health (MOH), using appropriate localized implementation models. Using a train-the-trainer model, local MHPs were trained by ParentCorps MHPs to provide $P D$ to teachers, reaching more than 300 teachers across Uganda and Nepal. In both countries we found that $P D$ provided by local MHPs led to greater use of evidence-based strategies by teachers and improved child mental health outcomes (see Supplemental file Table S1 for ParentCorps implementation models and effectiveness evidence in the US and global context). Our previous research showed the feasibility of a train-the-trainer model to $P D$ and a task-shifting approach to mental health promotion by teachers.

Building on this promising evidence from two LMICs (in both urban and rural contexts), the current study extends the existing partnership with the Ugandan $\mathrm{MOE}$ and $\mathrm{MOH}$ and further develops localized scalable and sustainable structure for $P D$ implementation in both rural and urban settings. This study investigates the effectiveness, underlying effectiveness-implementation mechanisms, and cost-effectiveness of $P D$ when implemented with a new scalable and sustainable model [30, 31].

\section{Considerations for a Scalable and Sustainable Model to Implement PD in Low Resource Contexts. To}

implement $P D$ in a scalable and sustainable manner, system- and workforce-level strategies need to be 
considered. Because the education system in Uganda does not have a school mental health service structure, and the majority of Ugandan schools do not have mental health resources, the provision of accessible and sustainable services to reach a large number of schools requires systems-level intervention. From a workforce sustainability and $\mathrm{EBI}$ service sustainment perspective, because $P D$ in LMICs relies on a task-shifting approach to provide the preventive service in schools, challenges related to task-shifting (i.e., teacher workforce stress and job burnout) and additional support strategies need to be considered. This study applies a scalable and sustainable implementation model, which integrates two system-level strategies and one workforce-level strategy (in addition to the task-shifting strategy described above that has been integrated into $P D$ ) that have been identified as effective for providing public health interventions at scale in low-resources settings.

A systems-level intervention strategy [30]. The Framework for Scaling Up proposed by the World Health Organization [30] provides a guiding framework to strategically carry out systems changes for scaling-up EBIs. Our implementation work is guided by this framework to address existing child mental health policy implementation and research gaps in Uganda. Starting in 2007, the Ugandan government proposed a series of reforms aimed at strengthening the country's mental health sectors [10,32]. Although several efforts have been made, no meaningful changes have occurred in child mental health [33]. To establish school-based mental health preventive intervention services, in 2013, our team initiated an effort to establish an implementation research partnership between US academic institutions and Ugandan academic, governmental, and NGO stakeholders. We carried out a series of child mental health epidemiological and intervention implementation studies to prepare for scale-up [26, 34, 35]. We systematically assessed local school ecological systems (e.g., policy, practices) and identified scale-up strategies that are in line with current policies and locally available resources [30, 36]. We developed a sustainable scale-up strategy promoted by the $\mathrm{MOE}$ and $\mathrm{MOH}$-train and build a workforce for $P D$ implementation embedded within the MOE teacher-training structure (i.e., teacher-training colleges) to provide $P D$ to teachers.

A partnership strategy [37]. Cross-disciplinary and cross-agency partnership strategies can be effective to overcome systems barriers when existing structures do not have sufficient capacity for large-scale public health program implementation [36, 38, 39]. In Uganda, Teacher Training Colleges (TTCs) are core institutions that provide in-service training for teachers. Therefore, they can serve as the key partner to provide the proposed PD. Most TTCs (96\%) are owned and funded by the government, and all TTCs are monitored and supported by the MOE [40]. Although the MOE has recommended a holistic approach to improve teacher competencies, including strategies for promoting child mental health, such training is underdeveloped in the current system. The Principal Medical Officer at the $\mathrm{MOH}$ is in charge of mental health services and is responsible for overseeing public education and mental health programs across the country. Most formal collaborations between governmental agencies for mental health services and those responsible for primary/community health focus on adults. Because of a limited number of MHPs in the country and lack of child mental health training in TTCs, a formal collaboration among $\mathrm{MOH}, \mathrm{MOE}$, TTCs, and MHP training institutions (e.g., Department of Psychiatry in Medical Schools) and teachers to task-shift and task-share preventive care tasks has the potential to create a sustainable structure to train 
and support teachers to promote child mental health[41]. The proposed study formalizes the role and structure of an implementation partnership across teacher education and mental health training institutions and the governmental sectors (i.e., $\mathrm{MOE}$ and $\mathrm{MOH}$ ) that has the potential to be sustainable over time.

A teacher workforce support strategy. Teachers in Uganda and other LMICs are vulnerable to job burnout and stress [42]. Data from our prior work in Uganda revealed that $79 \%$ of Ugandan teachers reported significant burden of stress (e.g., $70 \%$ workload stress, $41 \%$ job effectiveness, $23 \%$ emotional distress, $9 \%$ job dissatisfaction-related stress). Many Ugandan teachers requested stress management support from coaches to supplement $P D$. Teacher stress can result in anger, frustration, depression, exhaustion, and job ineffectiveness, and can have negative consequences for schools, teachers, students, and importantly EBI effectiveness $[43,44]$. Teachers suffering from stress and burnout may be less engaged in the $P D$, may have reduced motivation and ability to apply evidence-based strategies over time, or may experience difficulty in sustaining the added responsibility resulting from task-shifting. This effectivenessimplementation study tests a brief stress-management intervention (Teacher-Wellness or T-Wellness), as a complement to $P D$ (described below), and examines the underlying mechanisms through which teacher stress and stress management may facilitate or hinder the effectiveness, uptake and sustainment of evidence-based practices.

\section{Effectiveness-Implementation Aims and Hypotheses}

This study advances the science of implementation and intervention effectiveness in child mental health in LMICs by investigating the effectiveness-implemenation of $P D$ in urban and rural regions in Uganda when the $E B I$ is implemented using a scalable model. Given that a high percentage of Ugandan teachers experience occupational stress that may threaten $P D$ uptake, effectiveness, and sustainment, we also test a teacher stress management package, T-Wellness, as an enhancement to $P D$. The proposed protocol applies a Hybrid-Type II effectiveness-implementation 3-arm cluster-randomized controlled trial (cRCT) [45] to study the effectiveness-implementation of PD+T-Wellness (PDT) and PD alone (PD) relative to control. Three specific aims are as follows:

\section{To examine the short- and longer-term effectiveness of $P D$ and $P D T$ on teacher evidence-based} practices and child mental health outcomes when the EBI is implemented using a scalable model. Hypotheses: (a) PD is more effective than control; and (b) PDT is more effective than PD alone; and (c) $P D T$ has a more favorable cost-effectiveness ratio than $P D$

\section{To examine effectiveness mechanisms and theory of change underlying $P D$ and PDT. Hypotheses:} The mediational mechanisms of $P D$ and $P D T$ will be supported. Specifically, $P D$ will result in greater use of evidence-based practices by teachers and this will in turn result in improved child outcomes. $P D T$ will increase teacher stress management and evidence-based practices and together these will result in improved child outcomes.

3. To examine the implementation contextual factors and moderation mechanisms that contribute to teachers' uptake and sustainment of evidence-based practices within PD and PDT schools. We will 
assess the impact of implementation contextual factors (e.g., fidelity, teamwork alliance, leadership support climate) on teachers' PD/PDT implementation outcomes. Hypotheses: Better fidelity, teamwork alliance and more supportive contexts will be associated with better teacher uptake and sustainment of EBI practices.

\section{Theory of Change}

The Theory of Change is shown in Figure 1. The theory posits that $P D$ will promote teachers' use of evidence-based practices, and the impact of $P D$ on distal child mental health outcomes will be mediated primarily through these practices. Similarly, we expect that $P D T$ will impact distal child outcomes through both teachers' stress management and evidence-based practices. We anticipate that PD or PDT will have immediate impact on child social and emotional skills and longer-term impact on mental health. Because $P D / P D T$ will be implemented in diverse contexts, our theory of change also considers potential moderators from the Consolidated Framework for Implementation Research (CFIR) [46] -individual, intervention implementation, and school internal and external contexts.

\section{Methods}

\section{Trial Design}

We employ a matched-pair cRCT design and mixed methods evaluation data collection. For reporting, we follow the Consolidated Standards of Reporting Trials (CONSORT) for CRCT designs [47] and the Standard Protocol Items: Recommendations for Interventional Trials (SPIRIT) guidelines [48]. We conduct a Hybrid Type II effectiveness-implementation trial [45] in two regions in Uganda. The school is the unit of randomization because the program is applied at the school-level and builds a "school community" of teachers to promote student mental health. The three-arm cRCT design allows us to simultaneously test $P D$ effectiveness and study the added value of the T-Wellness to address teacher stress, which is a critical challenge to practical task-shifting effectiveness and sustainment. In addition, the Hybrid design, which considers CFIR domains of implementation contexts (listed in Figure 1), allows us to rigorously study other EBI effectiveness-implementation mechanisms, which can further inform decisions about optimal deployment and the generalizability of impact, and may accelerate the introduction of other valuable innovations into practice [45]. To have a more comprehensive understanding of possible underlying implementation and effectiveness mechanisms, we also plan to conduct qualitative interviews and focus groups, which will purposefully select $P D / P D T$ trainers, teachers, and parents. The study has been approved by the Institutional Review Boards of New York University Grossman School of Medicine (i2000117), Makerere University (REC REF 2020-143), and Uganda National Science and Technology (HS1057ES).

\section{Participants}

School recruitment and randomization. Primary schools in targeted Kibuli (urban) and Hoima (rural) districts will be identified from governmental school lists. These districts were selected based on MOE 
leaders' assessment of high need. To ensure approximately similar school characteristics in three conditions across two geographic regions, a stratified-block randomization procedure will be applied (Figure 2) [49]. A statistician who is unfamiliar with study schools will first match the schools on school size (teacher/student numbers) and school quality/performance (based on MOE data) within regions to ensure similar characteristics in urban and rural regions. Eighteen schools in 6 matched blocks (with blocks of size 3) from each region will be selected. Principals will be invited to attend information sessions hosted by the Ugandan study team During recruitment sessions, principals will be provided with details of study requirements and intervention implementation procedures. They will have an opportunity to ask questions and also complete a questionnaire on school demographics, commitment, willingness to facilitate data collection, and ability to allocate staff time to participate in the study. School principals who express interest and agree to allow teachers' voluntary participation will be eligible and will be consented. A total of 36 schools will be included for the effectiveness study. Computer-generated random numbers will be used to decide the randomization allocation sequence. Within each block (of size 3 ), one school will be randomly assigned to $P D T$, one to $P D$, and one to Control (receiving child mental health materials approved by $\mathrm{MOH} / \mathrm{MOE}$ ). The intervention and evaluation activities will be carried out in two consecutive cohorts using the two-cohort approach because it allows for building the capacity of TTC trainers to carry out $P D$ in a real-world context and provides time for TTC trainers to develop practice competency. Cohort 1 schools ( $n=18 ; 9$ urban and 9 rural) will start in 2021, and Cohort 2 schools $(n=18)$ in 2022. Schools from both cohorts will be actively involved for 2 years.

Teacher recruitment. All pre-primary and primary grade 1 to 4 teachers and teaching assistants, serving students between the ages of 3 and 10 years, will be eligible to participate. We include multiple grades because teachers in Uganda teach a wider age range of classes. Teachers' participation will be completely voluntary, with no consequence for opting out. Based on the $100 \%$ enrollment record from our prior $\mathrm{CRCT}$, we anticipate that nearly all eligible teachers will sign up for the study. We anticipate that 540 teachers from 36 schools will participate in the evaluation; and 360 intervention school teachers will receive $P D$ (180 with and 180 without T-Wellness). Based on enrollment from our prior study in Uganda, we anticipate that nearly all teachers will sign up for $P D$ (i.e., $>90 \%$ will participate in $P D$ ). Based on feedback from stakeholders, we will also recruit two parents from the Parent-Teacher-Associations (PTAs) in intervention schools to be part of the school-based team to support teachers to facilitate parent involvement (e.g., through sharing evidence-based knowledge and parenting strategies with families during parent-teacher conferences).

Parents and child recruitment. Students attending pre-primary and primary grades 1 to 4 (ages 3-10 years) and their parents/primary caregivers will be eligible to participate in the study. Given the large numbers of students in schools, research staff will randomly select $10 \%$ of students and families from each school (based on student lists provided by schools) and complete assessments over two years [50, 51]. A total of 1,980 families from 36 schools (averaging 55 families/per school) will participate in the study. Teachers will be informed of the students randomly selected for participation in the assessments and asked to introduce the study staff to the selected families. Primary caregivers from the selected families will be invited to consent for interviews, and for research staff to carry out assessments with 
their child. Children with parent consent will be asked to assent to the study. Although we only evaluate a subset of the sample, an estimated 13,200 students across 24 intervention schools will be exposed to $P D$. Teachers and parents who participate in study, will receive a small incentive for their time.

Local PD implementers/trainers and MHP supervisors. A total of 8 Ugandan TTC trainers (4 from each TTC) and 4 MHP supervisors will be recruited and trained to implement and support PD. TTC trainers will be required to have a minimum of university level of education and two or more years of experience in teacher training. MHP supervisors will be clinical psychologists, or mental health counselors with at least master's degrees and 2 years of experience, or psychiatric nurses (with at least bachelor's degree and 5 years of clinical experience). They will be recruited from local universities or mental health facilities. TTC trainers and MHPs who agree to participate will be asked to provide written informed consent, which will allow the research team to gather fidelity and competency data (self-reported, audio, or observational data) with their assistance.

Subsample for the Qualitative Study. Subsamples of study participants from the intervention schools will be selected to participate in qualitative interviews or focus groups aimed at better understanding the underlying mechanisms for the effective implementation and sustainment. For each study cohort we will carry out interviews and focus groups with $P D / P D T$ trainers $(\mathrm{n}=8)$, teachers and parents $(\mathrm{n}=40 ; 20$ from $P D$ and 20 from $P D T$ schools across urban and rural sites). Qualitative data will be conducted twice (post $P D / P D T$ intervention and a year after the intervention).

\section{Sample Size and Power}

We conducted power analyses for child and teacher effectiveness outcomes, assuming an intention-totreat (ITT) analysis. The power calculation is estimated primarily based on: i) the statistical analysis approach planned for this study (linear mixed effect models [52]); ii) the expected magnitude of the effects for the primary child and teacher outcomes from our prior Ugandan $P D$ study (i.e., child mental health $d=.39$; child social emotion competency $d=1.08$; teacher practice outcomes-observed $d=.55$ and self-report $d=.32$ ); and iii) detectable effects with $80 \%$ power of two-sided significance tests with $a=.05$. In CRCT designs, the detectable effect sizes depend on the usual study design parameters, as well as the cluster size, $\mathrm{N}$, and the cluster effect, i.e., the intra-cluster correlation coefficient (ICC). Detectable effects also depend on the test used (e.g., a test that accounts for baseline outcome or examines effectiveness with repeated observations is more powerful) [52]. We anticipate that 540 teachers (or 180 per intervention condition) and 1,980 parent-child pairs (or 660 per intervention condition) from 36 schools will participate in the study. We estimate power (for detectable effect size) based on the total sample (1,320 families and 360 teachers for two comparison conditions [ 1 intervention and 1 control]), as well as based on the sample from one region (660 families and 180 teachers for two conditions) with one or two post-intervention outcome evaluations, and assuming $20 \%$ loss of sample by Time 3 . Table $S 2$ in the Supplemental file gives the detectable effects for a range of cluster/ICC values and multiple scenarios. In the most conservative scenarios when the ICC $=0.05$, for 24 school clusters (2-condition comparisons across two regions), the detectable effects are $d=.14-.22$ for child outcomes, $d=.18-.38$ for teacher 
intermediate outcomes; and for 12 school clusters (2-condition comparisons in one region), the detectable effects are $d=.19-.42$ for child outcomes, $d=.25-.55$ for teacher intermediate outcomes. This study will have sufficient power to detect impacts that are meaningful and realistically achievable.

\section{Description of Intervention and Scalable Implementation Approach}

The PD Implementation Approach. The approach to ParentCorps PD implementation relies on Train-theTrainer (TTT) and a dynamic multi-layered supervision model [53]. As shown in Figure 3, an experienced clinical team from the ParentCorps Central Office in the US provides comprehensive virtual training and ongoing supervision to four Ugandan MHPs, including two who were previously trained and participated in the previous ParentCorps $P D$ study. The four-person Ugandan clinical team will oversee local implementation efforts including supporting and supervising the TTC Ugandan $P D$ trainers/facilitators. The ParentCorps clinical team from the Central Office in the US will provide virtual training to 8 TTC Ugandan $P D$ facilitators (32 hours over 8 half days) and the Ugandan clinical team will provide live ongoing supervision. Over a two month period ( 8 weekly meetings) working with the Ugandan clinical team, the TTC Ugandan PD facilitators will practice, receive feedback and refine aspects of $P D$ for the local context prior to the first round of implementation. Finally, with ongoing supervision from the Ugandan clinical teams (8 live group supervision sessions), the Ugandan TTC facilitators will provide ParentCorps $P D$ (21 hours over 3 days) to the Ugandan primary school teachers and 8 one-hour of coaching session over a 2-3 month period.

Teacher-Wellness Implementation Approach. T-Wellness was co-developed by US and Ugandan investigators, adapted from evidence-supported strategies for teacher stress and burnout management $[54,55]$. T-Wellness is a one-day workshop and three group-support sessions (45-60 minutes each) for teachers. MHPs from the Ugandan clinical team will be trained by study investigators to facilitate the workshop and the support sessions to teachers. Teachers from schools assigned to the PDT condition will receive the 1-day workshop right after receiving $P D$. The three group-support sessions will be integrated in the $P D$ coaching sessions. For implementation quality assurance, the Ugandan clinical team will receive supervision from the study investigators after each group-support session they provide to teachers.

\section{Intervention Conditions}

$P D$. The 3 days of ParentCorps $P D$ aims to help teachers to foster child social emotional learning, strong family-school relationships and safe, nurturing, and predictable classroom environments. There are four elements that the ParentCorps theory of action specifies as essential processes through which $P D$ strengthens teachers' use of evidence-based practices: building authentic relationships, honoring culture, translating the science of early childhood development, and practicing self-reflection. These essential elements are measurable aspects of the quality of facilitation that complement measures of fidelity to the manuals in explaining the extent to which the program targets change. Specifically, high quality, high fidelity facilitation is hypothesized to support teachers in developing increased capacity as defined by (1) 
knowledge of evidence-based strategies; (2) awareness of self and child; (3) intentional and responsive interactions; and (4) problem-solving and support-seeking as needed.

(see Table S1 in the Supplemental file for additional contents information).

$P D T$. The 1-day workshop and 3 group support sessions aim to increase teachers' self-awareness of their stress and regulation/coping styles, and support teachers to manage stress through practicing evidencebased strategies. Prior to the workshop, teachers will be asked to complete a stress and wellness selfassessment survey using a digital toolkit. A tailored report is generated right after the assessment to share with teachers to promote self-awareness and motivation for change. During the one-day workshop, four key topic areas will be covered: (i) understanding stress and job burden; (ii) self-appraisal and identification of areas for improvement; (iii) cognitive and behavioral strategies; and (iv) teacher-toteacher support and other additional resources. The group support sessions are to help teachers apply strategies to work toward their wellness goals.

Control Group. Teachers in control schools will receive mental health knowledge and promotion materials. In the second year of participation (after completion of the effectiveness evaluation), control schools will receive a one-day T-Wellness workshop (without $P D$ ) and 3 follow-up group-support sessions.

\section{Study Measures}

The evaluation design is guided by the implementation outcome framework [56]. The quantitative evaluation measures for teacher and child effectiveness outcomes will be assessed using multiple sources (data collected from objective classroom observation, parents, teachers, and children) and across 3 time points (T1 baseline, before PD; T2 immediately after the PD/PTD, about 3-4 months after T1; and T3, 9-12 months after T2). Research staff responsible for family and observation data collection will be masked to intervention conditions. To ensure masking, we will have an independent assessment team (led by a separate research coordinator), and members will not participate in any intervention activities. We will also train the implementation team on protocol to prevent unblinding. Table 1 lists the measures included in the study. Most of the measures have been used and validated in our previous pilot trial [26].

\section{EffectivenessOutcome Measures}

Child effectiveness outcomes. The primary outcome is child mental health (externalizing and internalizing problems), and the secondary outcome is social-emotional competency (emotion regulation, executive functioning). Parent and child report data will be gathered. Parents of study students will be interviewed by trained research staff (using English or Luganda, lasting 30-45 minutes). Participating children will be assessed by trained research staff in schools (lasting about 20-25 minutes).

Teacher effectiveness outcomes. The primary teacher outcomes are (i) EBI strategy use, which will be based on objective observation by an independent observation team (primary data source) and teacher report (secondary data source), and (ii) teacher perceived stress and stress management (teacher report). 
The secondary teacher outcome will be school-home connection, teacher-family relationship, and studentteacher relationship based on parent and student report.

\section{Implementation Outcome Measures}

Fidelity will be measured to assess the quality of implementation. Four fidelity measures will be considered, including adherence (the extent to which the TTC facilitators deliver the core intervention content and as per program guidelines), quality of program implementation (assessed based on teacher rating of their training expeirence of Coaches' competence (knowledge, preparation, ability to control discussion, enjoyable); engagement (assess trainees' level of $P D$ knowledge improvement from pre- to post-training; and exposure (measured by trainees' attendance in $P D$ and coaching sessions) [26]. Acceptability and Appropriateness of PD/PDT will also be measured based on teacher report and be assessed post-training and at T2 (after completing the full cycle of $P D / P D T$ ) [57].

Cost Measures. Costs will be measured using an activity-based micro-costing approach [58] in the intervention and control clusters (school), and in the extended implementation phase again in all clusters from 6 through 12 months ( $n=36$ clusters). Micro-costing entails a three-step approach where we identify, measure, and value resource use for all activities in each study arm. Resource use and cost data will be collected prospectively alongside the trial. All research costs will be excluded. Cost data collection will utilize standardized cost extraction forms and procedures that have been validated in our team's previous work in Uganda and other LMICs [59-64]. Prior to use, these tools will be tailored and customized to the $P D / P D T$ context. All costs will be adjusted for inflation, discounted to the intervention start year, and presented in US dollars.

\section{Contextual Moderators}

Constructs in CFIR domains will be measured to study moderation effect on teacher EBI strategy use outcomes. Selection of CFIR moderators is guided by factors identified in the literature as influential factors for implementation and effectiveness outcomes [46, 65]. Inner setting will include school structural and climate characteristics (classroom size, learning climate, leadership engagement, teamwork alliance); outer setting will include region (urban/rural); process will include partnership quality, fidelity, and cohort ( $1^{\text {st }}$ or $2^{\text {nd }}$ implementation cohort); intervention characteristics will include perceived $P D / P D T$ acceptability and appropriateness; and Individual teacher characteristics will include teacher years of experience and gender.

\section{Qualitative Assessment}

To have a more comprehensive understanding of possible mechanisms, we will conduct qualitative interviews and focus groups. Interview guides will comprise semi-structured questions relating to experiences with $P D / P D T$ and sustainment of $P D / P D T$. Participants will be asked to provide a narrative account of partnership approaches and efforts to implement and sustain $P D / P D T$, including barriers and facilitators experienced. Qualitative assessment will be conducted twice at T2 and T3. We will also 
conduct qualitative assessment separately for each cohort, which allows a better understanding of cohort effects and whether quality of implementation improves over time.

\section{Data Management}

All data will be managed and stored in REDCap (Research Electronic Data Capture). REDCap is a secure web application for building and managing surveys and databases for research studies, originally developed at Vanderbilt (www.project-redcap.org) with collaboration from a consortium of worldwide institutional partners. It provides automated export procedures for seamless data downloads to common statistical packages such as Excel, SPSS, SAS, Stata, and R. Access to study data in REDCap will be restricted to the specific members of the study team with authentication. Qualtrics will also be used when collecting data in the field through the offline mobile app function. When using Qualtrics offline mobile app, no identifying information will be collected. Qualtrics mobile app uses Transport Layer Security (TLS) encryption (also known as HTTPS), and data entered into the mobile app cannot be re-accessed in the front-end. Only selected staff members with have access to the data in the back-end through password protected accounts. Data will be entered using only the unique study identification number. Qualtrics data will then be transfer backed into REDCap as our database management system. All final study files for analyses will be captured and finalized ensuring that no personal identifiable information (PII), including students', parents' or teachers' names and contact information, are included. Electronic data entered that include contact identifying information (e.g., master list of consenting information, contact information/address) will be securely saved, and will not be linked to the study data. There will be additional levels of protection and access restrictions to this information.

\section{Data Analyses and Statistical Methods}

Preliminary Analyses. Prior to any outcome analyses, we will generate summary statistics for all data, summarizing with means and standard deviations for continuous variables and frequencies for categorical variables. Baseline equivalence between intervention and control schools will be examined. For measures that evaluate similar constructs, composite scales will be created (to minimize number of analyses). In addition, the distribution of study variables and missing data patterns will be inspected. For participants with partially missing data, a multiple imputation strategy using a Markov chain Monte Carlo approach will be applied. We will also sequentially impute data for each wave using predictive mean matching method separately for intervention and control groups to account for the possibility of different missing data patterns by condition [83-85]. Ten data sets will be imputed, and SAS PROC MIANALYZE will be used to combine the results for the final inference [86].

Analyses for Aim 1. To estimate effectiveness, we will apply intention-to-treat (ITT) analyses and first focus on between-subject comparisons of intervention vs. control (comparing $P D$ to control, comparing $P D T$ to control, and comparing $P D$ to $P D T$ ). We will estimate the impact of $P D$ on children and teachers post-intervention (T2, 4-5 months after T1) and at one-year follow up (T3, 12 months after T1). School and class nesting will be considered, and a multiple imputation strategy will be applied to account for missing data. Linear mixed effect models [52], using SAS PROC MIXED[86], will be applied to examine 
short and longer-term impacts. We will first examine the immediate impact by modeling post-intervention outcomes (T2) as a function of intervention, adjusting for T1 outcome measures. The model accounts for correlation between subjects (within-school and -class) by including school- and classroom-level (when appropriate) random intercepts. Next, we will study longer-term effectiveness outcomes (T2 to T3) by applying growth curve models and using repeated assessments over time. In these growth models, we will add time-relevant parameters to the model above, including school-level random slopes associated with time. The post-baseline scores will be modeled as a linear function of time, intervention indicator, and intervention-by-time interactions, adjusting for T1 scores and cohort.

Cost-effectiveness analysis of $P D$ and $P D T$ implementation models will be examined using approaches that have been applied in previous school-based and parenting-based child mental health promotion research [87-90]. The analysis will center on incremental cost-effectiveness ratios (ICERs), where the numerator represents the cost difference between the intervention arms and the control, and the denominator represents the difference in average intervention effects. To that end, the cost-effectiveness analysis of the intervention will involve examining how much the $P D / P D T$ intervention costs to achieve a unit of effect relative to the control group. The effects of the intervention will be estimated using the effect sizes $d$ (standardized mean difference between intervention and control groups) using an ITT approach. For the effectiveness outcomes, we will use an effect size of 0.2-0.4 as a benchmark; this corresponds to a small to medium effect size according to Cohen [91]. We will compute the perparticipant cost per 0.2-0.4 SD change for each child effectiveness outcome. Reporting of the costeffectiveness analysis will follow the Consolidated Health Economic Evaluation Reporting Standards (CHEERS) [92].

Analyses for Aim 2.We will examine mediation mechanisms for $P D$ and $P D T$ separately. The analysis will be built on the Aim 1 Linear mixed effect models. For $P D$, we will examine whether impacts of $P D$ on children's mental health are mediated through improvement in teachers' EBI practices (primary). For PDT, we will examine whether impacts of $P D T$ on child mental health are mediated through improvement on teachers' EBI practices and stress management (in cognitive and behavioral domains). The intermediate teacher outcomes will be based on T1 and T2 data, and the child outcomes will be based on T2 and T3 data (to capture changes over time).

Analyses for Aim 3. To study effectiveness-implementation moderation mechanisms, we will test whether impacts of the intervention on teacher effectiveness and EBI practice sustainment outcomes is moderated by CFIR contextual factors. We will apply similar approaches as in Aim 1 and add the moderator and moderator-by-intervention interaction terms in the analysis. T2 implementation and T2 and T3 teacher effectiveness outcome data from the PD/PDT intervention samples will be utilized. Any significant moderators identified will suggest important factors to be intervened on in future implementation to enhance the uptake of evidence-based strategies by teachers, or improve the effectiveness of task-shifting. 
Qualitative data analyses. For the qualitative focus group data, we will apply qualitative analysis methods. Interview data will be transcribed and analyzed using Atlas.ti software. To better understand partnership/scalable approaches, coding will focus on themes related to the partnership development process, usefulness of partnership frameworks in formalizing processes, scalable strategies, intervention implementation barriers, teacher stress, and strategies for overcoming teacher stress and other practices (considering CFIR). These analyses will help identify facilitators and barriers for partnership and implementation for carrying out the effectiveness study. For effectiveness-implementation mechanisms, qualitative analysis will focus on themes related to implementation barriers, facilitators, and contextual factors and processes that influence teacher intermediate and child effectiveness outcomes. Coding of qualitative data will follow a constant comparative analysis approach, where data are analyzed for themes that reflect project aims, which are then confirmed by further data analysis, followed by a third review of the data to identify additional themes [93-95].

\section{Discussion}

This study is the next step to our previous pilot effectiveness-implementation research, and

furthers our collaboration with the $\mathrm{MOE}, \mathrm{MOH}, \mathrm{TTC}$, mental health training institutions, and teachers in Uganda to provide preventive care in schools. We utilize a task-shifting and task-sharing cross-sector partnership approach to study the effectiveness and cost-effectivenss of a potentially scalable and sustainable EBI implemenation model for promoting mental health and social-emotional competence among primary school students. We also investigate strategies to address a common task-shifting challenge (workforce stress/burnout) and examine whether the incorporation of a workforce stress management strategy can improve the effectiveness and sustainment of EBI practices. The costeffectiveness analysis will inform policy and implemenation decisions, which are critical for the scale-up and sustainability of EBIs in low-resource contexts. Our study builds on the increasing body of evidence on task-shifting approaches of mental health promotion, scale-up reserch, and workforce-related implementation strategy testing. The effectiveness-implemenation study is designed in partnership with the $\mathrm{MOH}$ and $\mathrm{MOE}$, which addresses local govenrmental needs and has the potential to affect policy change and inform school mental health system development. The EBI/PD applied in this study has been tested both in the US and in LMICs (Uganda and Nepal). This study will address numerous implemenation, scaling-up, and translational research gaps. The trial will be started in 2021 school year, which is unique in that the intervention will be conducted in the context of the COVID-19 pandemic. The baseline and evaluation data may inform the impacts of the COVID-19 on school staff, parents, and students. The intervention is likely to mitigate the crisis and negative impacts that the pandemic has on school communities. Lessons learned and shared by the US and Ugandan collaborators can illuminate the processes and complexities of scaling-up a population-wide approach for child mental health. Furthermore, the theory-guided implementation can inform the feasibility and the relevance of the theories to be applied in LMIC contexts. The knowledge gained from this study can be applied to guide other EBI dissemination and implementation efforts that utilize task-shifting/task-sharing/cross-sectoral collaboration strategies in LMICs. 


\section{Trial Status}

At the time of manuscript submission (May 2021), the trial study has not yet started. Baseline data collection is planned to commence in June 2021, and the intervention is planned to begin in June-July 2021. There is a possibility that the trial will be further delayed because of the COVID-19 pandemic.

\section{Abbreviations}

Low-and-middle income countries (LMICs); Evidence-based interventions (EBIs); Mental health professionals (MHPs); cluster randomized controlled trial (CRCT); ParentCorps-Professional Development $(P D) ;$ PD + T-Wellness (PDT); Ministry of Education (MOE); Ministry of Health (MOH); Teacher Training Colleges (TTCs); The Consolidated Framework for Implementation Research (CFIR); the Consolidated Standards of Reporting Trials (CONSORT); Standard Protocol Items: Recommendations for Interventional Trials (SPIRIT) guidelines; The Consolidated Health Economic Evaluation Reporting Standards (CHEERS).

\section{Declarations}

Ethnical Approval and Consent to participate. All research participants will be consented for participation. Children under 18 will be accent for participation. All study procedures were approved by the New York University School of Medicine: (i20-00117) and the in-country local IRBs in Uganda: Makerere University (\#RECREF 2020-143), and the Uganda National Council of Science and Technology (UNCST HS1057ES).

Consent for publication. Any results that will be published in academic journals or conferences will adhere to IRB guidelines.

Availability of data and materials. Trial data will be deposited to NIMH Data Archive NDA (website http://nda.nih.gov) as part of the funding agreement

Competing Interests. The authors declare that they have no competing interests.

Funding. The project is supported by National Institute of Mental Health R01MH122654-01

Authors' Contributions. KH conceived of the effectiveness-implementation study design and drafted the manuscript. JN made substantial contributions to study design, study coordination and critically reviewed the manuscript for important intellectual content. ENK was involved in conception and design of the implementation study and scalable implementation model. HS was involved in conception and design of the scalable implementation model. TD was involved in T-Wellness implementation strategy design, and YT was involved in cost-effectiveness evaluation design. HP was involved in effectiveness and effectiveness-implementation mechanism testing analytical design. LB was involved in implementation study and scale-up model design. All authors critically reviewed the manuscript for important intellectual content and approved the final manuscript. 
Acknowledgements. Funding for this study was provided by National Institutes of Mental Health (grant number: R01MH122654). We wish to acknowledge the participation of the Ugandan Teacher Training Colleges and schools.

Authors' Information. Department of Population Health, New York University Grossman School of Medicine, 227 East, $30^{\text {th }}$ St, .New York, USA. . College of Health Science, Makerere University, PO Box 7072, Kampala, Uganda. . Uganda Ministry of Education and Sports, Embassy House, PO Box 7063, Kampala, Uganda. . Uganda Ministry of Health, PO Box 7272, Plot 6 Lourdel Road, Kampala, Uganda. . Department of Psychology, Hunter College of the City University of New York, 695 Park Avenue, New York, USA. . College of Global Public Health, New York University, 708 Broadway, New York, USA

\section{References}

1. population ages $\mathbf{0}$-14 - Uganda https://data.worldbank.org/indicator/SP.POP.0014.TO.ZS? locations=UG.

2. Uganda country profile [https://data.unicef.org/country/uga/].

3. Huang K-Y, Abura G, Theise R, Nakigudde J. Parental depression and associations with parenting and children's physical and mental health in Sub-Saharan African settings. Child Psychiatry Hum Dev. 2017;48:517-27.

4. MOE-Uganda: Education and SPorts Sector Fact Sheet 2002-2016. In.: Ministry of Education and Sports, The Republic of Uganda; 2016.

5. Baumann AA, Powell BJ, Kohl PL, Tabak RG, Penalba V, Proctor EE, Domenech-Rodriguez MM, Cabassa LJ. Cultural Adaptation and Implementation of Evidence-Based Parent-Training: A Systematic Review and Critique of Guiding Evidence. Child Youth Serv Rev. 2015;53:113-20.

6. Gardner F, Montgomery P, Knerr W. Transporting Evidence-Based Parenting Programs for Child Problem Behavior (Age 3-10) Between Countries: Systematic Review and Meta-Analysis. J Clin Child Adolesc Psychol. 2016;45(6):749-62.

7. Huang KY, Nakigudde J, Rhule D, Gumikiriza-Onoria JL, Abura G, Kolawole B, Ndyanabangi S, Kim S, Seidman E, Ogedegbe G, et al. Transportability of an Evidence-Based Early Childhood Intervention in a Low-Income African Country: Results of a Cluster Randomized Controlled Study. Prev Sci. 2017;18(8):964-75.

\section{Education and Sports Sector Fact Sheet 2002-2016} http://www.education.go.ug/files/downloads/FACT\%20\%20\%20SHEET\%202016.pdf.

9. School. Enrollement, Primary (\% net) http://data.worldbank.org/indicator/SE.PRM.NENR.

10. Ssebunnya J, Kigozi F, Ndyanabangi S. Developing a national mental health policy: a case study from Uganda. PLoS Med. 2012;9:e1001319.

11. Brotman LM, Dawson-McClure S, Kamboukos D, Huang K-Y, Calzada E, Goldfeld K, Petkova E. Effects of ParentCorps in pre-kindergarten on child mental health and academic performance: follow-up of a randomized clinical trial through 8 years of age. JAMA Pediatrics. 2016;170:1-7. 
12. Webster-Stratton C, Reid MJ, Stoolmiller M. Preventing conduct problems and improving school readiness: evaluation of the incredible years teacher and child training programs in high-risk schools. J Child Psychol Psychiatry. 2008;49:471-88.

\section{Global School Health Initiative [https://.}

14. Lansford JE, Dodge KA. Cultural norms for adult corporal punishment of children and societal rates of endorsement and use of violence. Parenting: Science Practice. 2008;8(3):257-70.

15. Brotman LM, Calzada E, Huang K-Y, Kingston S, Dawson-McClure S, Kamboukos D, Rosenfelt A, Schwab A, Petkova E. Promoting effective parenting practices and preventing conduct problems among ethnic minority families from low-income, urban communities. Child Dev. 2011;82:258-76.

16. Calzada E, Huang K-Y, Anicama C, Fernandez Y, Brotman LM. Test of a cultural framework of parenting with Latino families of young children. Culture Diversity Ethnic Minority Psychology. 2012;18:285-96.

17. Huang K-Y, Calzada E, Kamboukos D, Rhule D, Cullen KW, Cheng S, Brotman LM. Applying public health frameworks to advance the promotion of mental health among Asian American children. Asian American Journal of Psychology. 2014;5:145-52.

18. Huang KY, Cheng S, Calzada E, Brotman LM. Symptoms of anxiety and associated risk and protective factors in young Asian American children. Child Psychiatry Human Development. 2012;43:761-74.

19. Lawire M. Parenting around the world: Plus ca Change. Journal of Family Studies Special Issue: Parenting around the world. 2009;15(3):204-6.

20. Bradley RH, Corwyn RF. Caring for children around the world: A view from HOME. international Journal of Behavioral Development. 2005;29(6):468-78.

21. Greenfield PM, Suzuki LK: Culture and human development: Implications for parenting, education, pediatrics, and mental health. In: Handbook of Child Psychology, Vol4. 5 edn. Edited by Sigel IE, Renninger KA. NY: Wiley; 1998: 1059-1107.

22. Brotman LM, Kingston S, Bat-Chava Y, Caldwell BM, Calzada E. Training school personnel to facilitate a family intervention to prevent conduct problems. Early Education Development. 2008;19(4):622-42.

23. Dawson-McClure S, Calzada E, Huang K-Y, Kamboukos D, Rhule D, Kolawole O, Petkova E, Brotman LM. A population-level approach to promoting healthy child development and school success in lowincome, urban neighborhoods: impact on parenting and child condut problems. Prev Sci. 2015;16:279-90.

24. Semrau M, Evans-Lacko S, Alem A, Ayuso-Mateos JL, Chisholm D, Gureje O, Hanlon C, Jordans M, Kigozi F, Lempp H, et al. Strengthening mental health systems in low- and middle-income countries: the Emerald programme. BMC Med. 2015;13:79.

25. Kigozi F, Ssebunnya J, Kizza D, Cooper S, Ndyanabangi S, the Mental Health and Poverty Project. An overview of Uganda's mental health care system: results from an assessment using the world health 
organization's assessment instrument for mental health system (WHO-AIMS). International Journal of Mental Health System. 2010;4(1):1-9.

26. Huang K-Y, Nakigudde J, Ruhule D, Gumikiriza JL, Abura G, Kolawole B, Ndyanabangi S, Kim S, Seidman E, Ogedegbe G, et al. Transportability of an Evidence-based Early Childhood Intervention in a Low-Income African Country: Results of a Cluster Randomized Controlled Implementation Study. Prev Sci. 2017;18:964-75.

27. Huang K-Y, Nakigudde J, Rana H, Castillo T. Implementing an Early Childhood School-Based Mental Health Preventive Intervention in Low-Resource Schools in Nepal and Uganda. In: NIMH/Grand Challenges Canada: Transformative Opportunities for Solving the Grand Challenges in Global Mental Health. Bethesta; 2017.

\section{Task shifting-Global Recommendation and Guidlines}

http://data.unaids.org/pub/Manual/2007/ttr_taskshifting_en.pdf.

\section{Task shifting to tackle health worker shortages}

http://www.who.int/healthsystems/task_shifting_booklet.pdf.

30. WHO: Nine steps for developing a sacling-up strategy. In. Geneva, Switzerland: WHO; 2010.

31. Chambers DA, Glasgow RE, Stange KC. The dynamic sustainability framework: Addressing the paradox of sustainment amied ongoing change. Implementation Science 2013, 8.

32. Uganda Ministry of Health. Child and Adolescent Mental Health Guideline In. Uganda Ministry of Health; 2013.

33. Ejuu G. The status of implementation of the education sector early childhood development policy in Uganda. In.: Uganda National Commission for UNESCO; 2012.

34. Huang K-Y, Nakigudde J, Brotman L: Use of task-shifting to scale-up child mental health services in low-resource Ugandan schools: Role of contextual factors on program implementation. Implementation Science 2015, 10 (Suppl 1).

35. Huang K-Y, Nakigudde J, Calzada E, Boivin M, Ogedegbe G, Brotman LM. Implementing an early childhood school-based mental health promotion intervention in low-resource Ugandan schools: Study protocol for a cluster randomiozed controlled trial. Trials. 2014;15:471.

36. Iwelunmor J, Blackstone S, Veira D, Nwaozuru U, Airhihenbuwa C, Munodawafa D, Kalipeni E, Jutal A, Shelley D, Ogedegebe G. Toward the sustainability of health interventions implemented in subSaharan Africa: a systematic review and conceptual framework. Implementation science: IS. 2016;11:43.

37. Huang K-Y, Kwon SC, Cheng S, Kamboukos D, Shelley D, Brotman LM, Kaplan SA, Olugbenga O, Hoagwood K. Unpacking Partnership, Engagement, and Collaboration Research to Inform Implementation Strategies Development: Theoretical Frameworks and Emerging Methodologies. Frontiers in public health. 2018;6:190.

38. Aguirre J, Carrion VG. Integrated behavioral health services: a collaborative care model for pediatric patients in a low-income setting. Clin Pediatr. 2013;52(12):1178-80. 
39. Aupont O, Doerfler L, Connor DF, Stille C, Tisminetzky M, McLaughlin TJ. A collaborative care model to improve access to pediatric mental health services. Adm Policy Ment Health. 2013;40(4):264-73.

40. Teacher Edudation http://www.education.go.ug/data/smenu/14/Teacher\%20Education.html.

41. WHO. Uganda MOH. WHO-AIMS report on mental health system in Uganda. In. Kampala, Uganda; 2006.

42. Ugwoke SC, Eseadi C, Onuigbo LN, Aye EN, Akaneme IN, Oboegbulem Al, Ezenwaji IO, Nwobi AU, Nwaubani OO, Ezegbe BN, et al. A rational-emotive stress management intervention for reducing job burnout and dysfunctional distress among special education teachers: An effect study. Medicine. 2018;97(17):e0475.

\section{Teacher Issues in Uganda: a shared vision for an effective teachers policy} http://unesdoc.unesco.org/images/0022/002297/229777e.pdf.

44. Adriaenssens J, De Gucht V, Maes S. Causes and consequences of occupational stress in emergency nurses, a longitudinal study. J Nurs Manag. 2015;23(3):346-58.

45. Curran GM, Bauer M, Mittman B, Pyne JM, Stetler C. Effectiveness implementation hybrid designs: combining elements of clinical effectiveness and implementation research to enhance public health impact. Med Care. 2012;50:217-26.

46. Damschroder LJ, Aron DC, Keith RE, Kirsh SR, Alexander JA, Lowery JC. Fostering implementation of health services research findings into practice: a consolidated framework for advancing implementation science. Implementation science: IS. 2009;4:50.

47. Hemming K, Taljaard M, McKenzie JE, Hooper R, Copas A, Thompson JA, Dixon-Woods M, Aldcroft A, Doussau A, Grayling $M$, et al. Reporting of stepped wedge cluster randomised trials: extension of the CONSORT 2010 statement with explanation and elaboration. BMJ. 2018;363:k1614.

48. Chan AW, Tetzlaff JM, Altman DG, Laupacis A, Gøtzsche PC, Krleža-Jerić K, Hróbjartsson A, Mann H, Dickersin K, Berlin JA, et al. SPIRIT 2013 statement: defining standard protocol items for clinical trials. Ann Intern Med. 2013;158(3):200-7.

49. Broglio K. Randomization in Clinical Trials: Permuted Blocks and Stratification. JAMA. 2018;319:2223-4.

50. Baker-Henningham H, Scott S, Jones K, Walker S. Reducing child conduct problems and promoting social skills in a middle-income country: Cluster randomised controlled trial. The British Journal of Psychiatry. 2012;201:101-8.

51. Webster-Stratton C, Reid MJ, Stoolmiller M. Preventing conduct problems and improving school readiness: evaluation of the Incredible Years Teacher and Child Training Programs in high-risk schools. Journal of Child Psychology Psychiatry. 2008;49(5):471-88.

52. Diggle PJ, Heagerty P, Liang K-Y, Zeger SL. Analysis of Longitudinal Data. New York: Oxford University Press; 2005.

53. Murray LK, Dorsey S, Bolton P, Jordans MJ, Rahman A, Bass J, Verdeli H. Building capacity in mental health interventions in low resource countries: an apprenticeship model for training local providers. International journal of mental health systems. 2011;5(1):30. 
54. Czabała C, Charzyńska K, Mroziak B. Psychosocial interventions in workplace mental health promotion: an overview. Health Promot Int. 2011;26(Suppl 1):i70-84.

55. Kangas-Dick K, O'Shaughnessy E. Interventions That Promote Resilience among Teachers: A Systematic Review of the Literature. International Journal of School Educational Psychology. 2020;8(2):131-46.

56. Proctor E, Silmere H, Raghavan R. al. e: Outcomes for implementation research: conceptual distinctions, measurement challenges, and research agenda. Adm Policy Ment Health. 2011;38:6576.

57. Weiner BJ, Lewis CC, Stanick C, Powell BJ, Dorsey CN, Clary AS, Boynton MH, Halko H. Psychometric assessment of three newly developed implementation outcome measures. Implementation science: IS. 2017;12(1):108.

58. Gold MR, Siegel JE, Russell LB, Weinstein MC. Cost-effectiveness in Health and Medicine. New York: Oxford University Press; 1996.

59. Opoku-Boateng YN, Kretchy IA, Aryeetey GC, Dwomoh D, Decker S, Agyemang AS, Tozan Y, Aikins M, Nonvignon J. Economic cost and quality of life of family caregivers of schizophrenic patients attending psychiatric hospitals in Ghana. BMC Health Serv Res. 2017;17:697.

60. Tozan Y, Headley TY, Sewe MO, Schwartz E, Shemesh T, Cramer JP, Eberhardt KA, Ramharter M, Harrison N, Leder K, et al: A Prospective Study on the Impact and Out-of-Pocket Costs of Dengue Illness in International Travelers. The American Journal of Tropical Medicine and Hygiene 2019.

61. Tozan Y, Ratanawong P, Sewe MO, Wilder-Smith A, Kittayapong P. Household costs of hospitalized dengue illness in semi-rural Thailand. PLOS Neglected Tropical Diseases. 2017;11:e0005961.

62. Liyanage P, Rocklöv J, Tissera H, Palihawadana P, Wilder-Smith A, Tozan Y. Evaluation of intensified dengue control measures with interrupted time series analysis in the Panadura Medical Officer of Health division in Sri Lanka: a case study and cost-effectiveness analysis. The Lancet Planetary Health. 2019;3:e211-8.

63. Thalagala N, Tissera H, Palihawadana P, Amarasinghe A, Ambagahawita A, Wilder-Smith A, Shepard DS, Tozan Y. Costs of Dengue Control Activities and Hospitalizations in the Public Health Sector during an Epidemic Year in Urban Sri Lank. PloS Negl Trop Dis. 2016;10:e0004466.

64. Tozan Y, Sun S, Capasso A, Shu-Huah Wang J, Neilands TB, Bahar OS, Damulira C, Ssewamala FM. Evaluation of a savings-led family-based economic empowerment intervention for AIDS-affected adolescents in Uganda: A four-year follow-up on efficacy and cost-effectiveness. PLoS One. 2019;14(12):e0226809.

65. Damschroder LJ, Lowery JC. Evaluation of a large-scale weight management program using the consolidated framework for implementation research (CFIR). Implementation science: IS. 2013;8:51.

66. Nakigudde J, Bauta B, Wolf S, Huang KY. Screening Child Social Emotional and Behavioral Functioning in Low-Income Country Contexts. Jacobs Journal of Psychiatry Behavioral Science. 2016;2:016.

67. PROMIS. PROMIS Pediatric Short Form v2.0- Anger 5a. In.; 2017. 
68. PROMIS. PROMIS Pediatric Item Bank v2.0 - Anxiety/Fear - Short Form 8a. In.; 2017.

69. PROMIS. PROMIS Pediatric Item Bank v2.0 - Depressive Symptoms - Short Form In.; 2018.

70. Fasfous AF, Peralta-Ramirez MI, Pérez-Marfil MN, Cruz-Quintana F, Catena-Martinez A, Pérez-García M. Reliability and validity of the Arabic version of the computerized Battery for Neuropsychological Evaluation of Children (BENCI). Child Neuropsychol. 2015;21:210-24.

71. Bernard ME. Teacher beliefs and stress. J Rat-Emo Cognitive-Behav Ther. 2016;34:209-24.

72. Connor-Smith JK, Compas BE, Wadsworth ME, Thomsen AH, Saltzman H. Responses to stress in adolescence: Measurement of coping and involuntary stress responses. Journal Of Consulting Clinical Psychology. 2000;68(6):976-92.

73. Yao S, Xiao J, Zhu X, Zhang C, Auerbach RP, McWhinnie CM, Abela JRZ, Wang C. Coping and Involuntary Responses to Stress in Chinese University Students: Psychometric Properties of the Responses to Stress Questionnaire. J Pers Assess. 2010;92(4):356-61.

74. Nakigudde J, Musisi S, Ehnvall A, Airaksinen E, Agren H. Adaptation of the multidimensional scale of perceived social support in a Ugandan setting. Afr Health Sci. 2009;9(Suppl 1(Suppl 1):35-41.

75. Victor SE, Klonsky ED. (2016).: Validation of a brief version of the Difficulties in Emotion Regulation Scale (DERS-18) in five samples. Journal of Psychopathology and Behavioral Assessment 2016, 38:582-589.

76. Family and Provider/Teacher Relationship Quality (FPTRQ) Parent Measure [https://.

77. McEwan PJ. Cost-effectiveness analysis of education and health interventions in developing countries. Journal of Development Effectiveness. 2012;4(2):189-213.

78. Walker TJ, Rodriguez SA, Vernon SW, Savas LS, Frost EL, Fernandez ME. Validity and reliability of measures to assess constructs from the inner setting domain of the consolidated framework for implementation research in a pediatric clinic network implementing HPV programs. BMC Health Serv Res. 2019;19(1):205.

79. Organizational. Readiness for Change http://ibr.tcu.edu/forms/organizational-staff-assessments/.

80. Huijg JM, Gebhardt WA, Dusseldorp E, Verheijden MW, van der Zouwe N, Middelkoop BJ, Crone MR. Measuring determinants of implementation behavior: psychometric properties of a questionnaire based on the theoretical domains framework. Implementation science: IS. 2014;9:33.

81. Akena D, Joska J, Obuku EA, Stein DJ. Sensitivity and specificity of clinician administered screening instruments in detecting depression among HIV-positive individuals in Uganda. AIDS Care 2013, 25.

82. Kroenke K, Spitzer R, Williams JB. The PHQ-9 validity of a brief depression severity measure. J Gen Intern Med. 2001;16:606-13.

83. Schenker N, Taylor JMG. Partially parametric techniques for multiple imputation. Comp Stat Data An. 1996;22(4):425-46.

84. Heitjan DF, Roderick JAL. Multiple Imputation for the Fatal Accident Reporting System. J Roy Statist Soc Ser C. 1991;40(1):13-29.

85. Little R, Rubin D. Statistical analysis with missing data. New Jersey: John Wiley and Sons, Inc.; 2002. 
86. SAS Institute Inc. Base SAS ® 9.3 Procedures Guide. Cary, NC: Copyright @ 2011. SAS Institute Inc.; 2011.

87. Tozan Y, Eili Y, Klein SD, Rajashree P, Ramanan L, Joel GB. Pre-referral rectal artesunate for treatment of severe childhood malaria: a cost-effectiveness analysis. The Lancet. 2010;379:1910-5.

88. Tozan Y, Joel N, James OW: Improving rural health and eliminating extreme poverty: A case study on the Millennium Villages Project." In:, (eds).. In: Social determinants approaches to public health: from concept to practice edn. Edited by Blas E, Sommerfeld J, Kurup AS. Geneva: World Health Organization 2011.

89. Tozan Y, Albrecht J, Olaf M. Evaluation of the Affordable Medicines Facility - malaria. The Lancet. 2013;381:1095.

90. Tozan Y, Ratanawong P, Louis VR, Kittayapong P, Wilder-Smith A. Use of insecticide-treated school uniforms for prevention of dengue in schoolchildren: a cost-effectiveness analysis. PLoS One. 2014;9(9):e108017.

91. Cohen J. Statistical power analysis for the behavioral sciences. 2 ed. Hillsdale: Lawrence Erlbaum Associates; 1988.

92. Husereau D, Drummond M, Petrou S, Carswell C, Moher D, Greenberg D, Augustovski F, Briggs AH, Mauskopf J, Loder E. Consolidated Health Economic Evaluation Reporting Standards (CHEERS)explanation and elaboration: a report of the ISPOR Health Economic Evaluation Publication Guidelines Good Reporting Practices Task Force. Value in health: the journal of the International Society for Pharmacoeconomics Outcomes Research. 2013;16(2):231-50.

93. Glaser BG, Strauss AL. The discovery of grounded theory: Strategies for qualitative research. Chicago: Aldine Publishing Co.; 1967.

94. Padgett DL. Qualitative Methods in Social Work Research. London: Sage; 1998.

95. Padgett DK: Qualitative and mixed methods in public health Sage; 2012.

96. Huang K-Y, Nakigudde J, Rhule D, Gumikiriza-Onoria JL, Abura G, Kolawole B, Ndyanabangi S, Kim S, Seidman E, Ogedegbe G, et al: Transportability of an Evidence-Based Early Childhood Intervention in a Low-Income African Country: Results of a Cluster Randomized Controlled Study. Prevention Science 2017.

97. Dawson-McClure S, Calzada E, Huang K-Y, Kamboukos D, Rhule D, Kolawole O, Petkova E, Brotman LM. A population-level approach to promoting healthy child development and school success in lowincome, urban neighborhoods: impact on parenting and child condut problems. Prev Sci. 2014;16:279-90.

\section{Tables}

Table 1. Key Study Measures for Effectiveness-Implementation Study: Constructs and Measures 


\section{Dimensions \\ Constructs \\ Measures \& Sources of Data}

(Assessment

Time)

\section{Child Effectiveness Outcomes}

$(T 1, T 2, T 3)$

\author{
Mental Health \\ (Primary): \\ Exteranalizing \& \\ Internalizing
}

Social Emotional
Competency
(Secondary): Emotion
regulation;
Relationship;
Executieve
functioning

Strengths \& Difficulties Questionnaire $(\mathrm{a}=.63-.80)[66](\mathrm{P})$; PROMIS Anger ( $(\mathrm{a}=.78)$, Anxiety/Fear $(\mathrm{a}=.90)$, Depression $(a=.87)[67-69](P)$

Emotion-regulation $(\mathrm{a}=.90)[26](\mathrm{P}) ;$ \& Peer relationship $\mathrm{a}=.75)[26](P)$; Comprehensive Computerized Battery for Child Psychological Assessment (for executive functioning $[70](C)$

\section{Teacher Effectiveness Outcomes}

$(T 1, T 2, T 3)$

\section{Teacher EBI Practice (Primary): PD} strategies use

\section{Teacher Stress \& Management (Primary)}

Teacher EBI Practices Classroom Observation ( $\mathrm{a}=.68-.72)$ [26] (0); EBI Strategies Practice Questionnaire ( $\mathrm{a}=.69-.80)$ [26] (T)

Stress Questionnaire [71] (T); Responses to Stress Questionnaire [72, 73] (T); Social Support $(a=.97)[74](T)$. Difficulties in Emotion Regulation Scale (DERS-18) [75] $(\mathrm{T})$

\section{Social Engagement} (Secondary): Parent \& student engagement

\section{Implementation Outcomes}

(during

Training and Coaching sessions)

Fidelity: (1)

Adherence; (2)

Quality of implementation; (3)

Engagement (preand post-training knowledge gain) (4) Exposure

\section{Appropriateness \& Usefulness of PD/PDT}

\section{PD/PDT} implementation costs

\section{Contextual Moderators- in CFIR domains}

(T1/beginning of the PD/PDT)
Inner setting: (1)

School structural characteristics; (2) School climate (culture, learning climate, leadership engagement, team work alliance)

Individual Teacher characteristics: Role
Teacher-student relationship (C)( $\mathrm{a}=$.78)[27]; Family and Teacher Relationship Quality[76](P)

(1) PD/PDT Fidelity Checklists (F)[26]: after training \& coaching session; (2) Teacher Training Experience Rating: facilitator competency (knowledge, preparation, ability to control discussion, enjoyable) (rating after training and coaching)( $(\mathrm{a}=.72)[26](\mathrm{T})$; (3) EB/ Strategy Knowledge (test-retest $\mathrm{r}=.35-.43)[26](\mathrm{T}) ;(4)$ Attendance tracking[26] (F)

Implementation outcome measure [57] (T)
Implementation costs for PD/PDT: actual program costs (with \& without monitoring cost)[77]

(1) School structural characteristics (classroom size); (2) School Environment \& Climate Survey (adapted from Inner-setting scale [78] and Organization Climate Questionnaire ( $\mathrm{a}=.65-.85)$ [79] (T) 
clarity; Self-efficacy;

Mental health

Intervention

characteristics:

See implementation outcome measures above

Acceptability,

Appropriateness,

Processes:

Partnership Quality Scale (Coach-teacher relationships,

Partnership quality,

Fidelity, Intervention

support from coaches, support from teachers)[37](T)

cohort $\left(1^{\text {st }}\right.$ or $2^{\text {nd }}$

cohort)

Outer setting:

Urban/rural region

Geographic region (urban/rural)

Note. For child effectiveness outcomes, primary data source is parent-report (P); and secondary data source is child-report/testing (C). For teacher effectiveness outcomes, primary data sources is classroom observation ( $(0)$ \& teacher report $(\mathrm{T})$; and secondary data sourcs are Parent $(\mathrm{P})$ and child report (C). CFIR contextual data will be gathered from from Training/coaching session tracking data from facilitator report $(F)$ and teachers-report $(T)$.

\section{Figures}

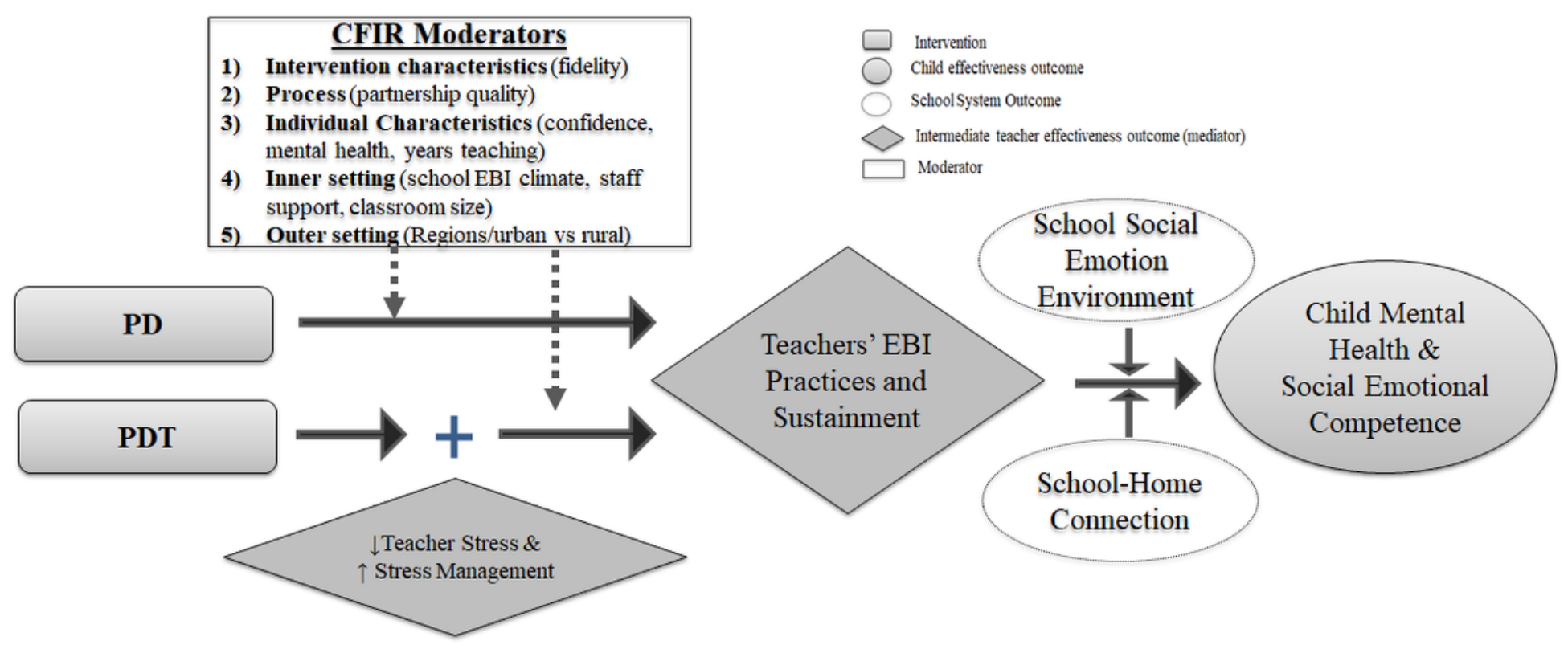

Figure 1

Hypothesized mechanisms for PD and PDT 


\begin{tabular}{|c|c|c|c|c|c|c|}
\hline Approach & \multicolumn{6}{|c|}{$\begin{array}{l}\text { School Lists from Two District of Education Offices in Uganda } \\
\text { - Include mid to large size of school (mid size: } 600-900 \text { students; }>900 \text { students) } \\
\text { Create blocks of schools ( } 3 \text { in each block) that matched with size and performance. }\end{array}$} \\
\hline \multirow[t]{3}{*}{ Enrollment } & \multicolumn{6}{|c|}{$\begin{array}{l}\text { School Enrollment bv Geographic Region } \\
\text { ize and } 3 \text { blocks of large-size of schools from each region } \\
\text { schools reject, a random block will be selected again from the pool of school-blocks) } \\
\text { ocks) from each region (or } 36 \text { schools from } 12 \text { blocks across two regions) }\end{array}$} \\
\hline & \multicolumn{6}{|c|}{$\begin{array}{l}\text { Teacher \& Familv Enrollment from the Participation Schools } \\
\text { - Assess teacher and PTA eligibility, and recruit } 540 \text { teachers and } 24 \text { PTAs }(\sim 15 \text { teachers per school) } \\
\text { - Assess family eligibility \& recruitment } 1980 \text { parent-child pairs }(\sim 55 \text { parent-child pairs per school })\end{array}$} \\
\hline & \multicolumn{6}{|c|}{ Stratified according to Geographic Region } \\
\hline \multirow[t]{2}{*}{ Allocation } & \multicolumn{3}{|c|}{ Randomly assign 3 schools within each block to 3 arms } & \multicolumn{3}{|c|}{$\begin{array}{l}\text { Rural Region } \\
\text { Randomly assign } 3 \text { schools within each block to } 3 \mathrm{arms}\end{array}$} \\
\hline & $\begin{array}{l}\text { Allocated to } \\
\text { Group A } \\
\text { (n=6 schools) } \\
90 \text { teachers; } \\
6 \text { PTAs; } \\
330 \text { P-C pairs }\end{array}$ & $\begin{array}{l}\text { Allocated to } \\
\text { Group B } \\
\text { (n=6 schools) } \\
90 \text { teachers; } \\
6 \text { PTAs; } \\
\text { 330 P-C pairs }\end{array}$ & $\begin{array}{l}\text { Allocated to } \\
\text { Group C } \\
\text { (n=6 schools) } \\
90 \text { teachers; } \\
330 \text { P-C pairs }\end{array}$ & $\begin{array}{l}\text { Allocated to } \\
\text { Group A } \\
\text { (n=6 schools) } \\
90 \text { teachers; } \\
\text { 6 PTAs; } \\
330 \text { P-C pairs }\end{array}$ & $\begin{array}{l}\text { Allocated to } \\
\text { Group B } \\
\text { (n=6 schools) } \\
90 \text { teachers; } \\
6 \text { PTAs; } \\
330 \text { P-C pairs }\end{array}$ & $\begin{array}{l}\text { Allocated to } \\
\text { Group C } \\
\text { (n=6 schools) } \\
90 \text { teachers; } \\
330 \text { P-C pairs }\end{array}$ \\
\hline $\begin{array}{c}\text { Baseline } \\
\text { Assessment }\end{array}$ & $\begin{array}{l}\text { T1 Baseline } \\
\text { assessment }\end{array}$ & $\begin{array}{l}\text { T1 Baseline } \\
\text { assessment } \\
\end{array}$ & $\begin{array}{l}\text { T1 Baseline } \\
\text { assessment }\end{array}$ & $\begin{array}{l}\text { T1 Baseline } \\
\text { assessment }\end{array}$ & $\begin{array}{l}\text { T1 Baseline } \\
\text { assessment }\end{array}$ & $\begin{array}{l}\text { T1 Baseline } \\
\text { assessment }\end{array}$ \\
\hline Intervention & Receive $P D$ & Receive $P D T$ & Control & Receive $P D$ & Receive $P D T$ & Control \\
\hline \multirow[t]{2}{*}{ Follow-up } & $\begin{array}{c}\text { T2 Follow-up } \\
\text { assessment }\end{array}$ & $\begin{array}{c}\text { T2 Follow-up } \\
\text { assessment }\end{array}$ & $\begin{array}{c}\text { T2 Follow-up } \\
\text { assessment }\end{array}$ & $\begin{array}{c}\text { T2 Follow-up } \\
\text { assessment }\end{array}$ & $\begin{array}{c}\text { T2 Follow-up } \\
\text { assessment }\end{array}$ & $\begin{array}{c}\text { T2 Follow-up } \\
\text { assessment }\end{array}$ \\
\hline & $\begin{array}{c}\text { T3 Follow-up } \\
\text { assessment }\end{array}$ & $\begin{array}{l}\text { T3 Follow-up } \\
\text { assessment }\end{array}$ & $\begin{array}{l}\text { T3 Follow-up } \\
\text { assessment }\end{array}$ & $\begin{array}{c}\text { T3 Follow-up } \\
\text { assessment }\end{array}$ & $\begin{array}{c}\text { T3 Follow-up } \\
\text { assessment }\end{array}$ & $\begin{array}{c}\text { T3 Follow-up } \\
\text { assessment }\end{array}$ \\
\hline
\end{tabular}

Figure 2

Diagram of enrollment, randomization, and follow-up 
Receiving Training from ParentCorps Central Office

( $32 \mathrm{hrs} / \mathbf{8}$ half day) to learn the $P D$ and experiential and effective strategies to facilitate adult learning and skill acquisitions

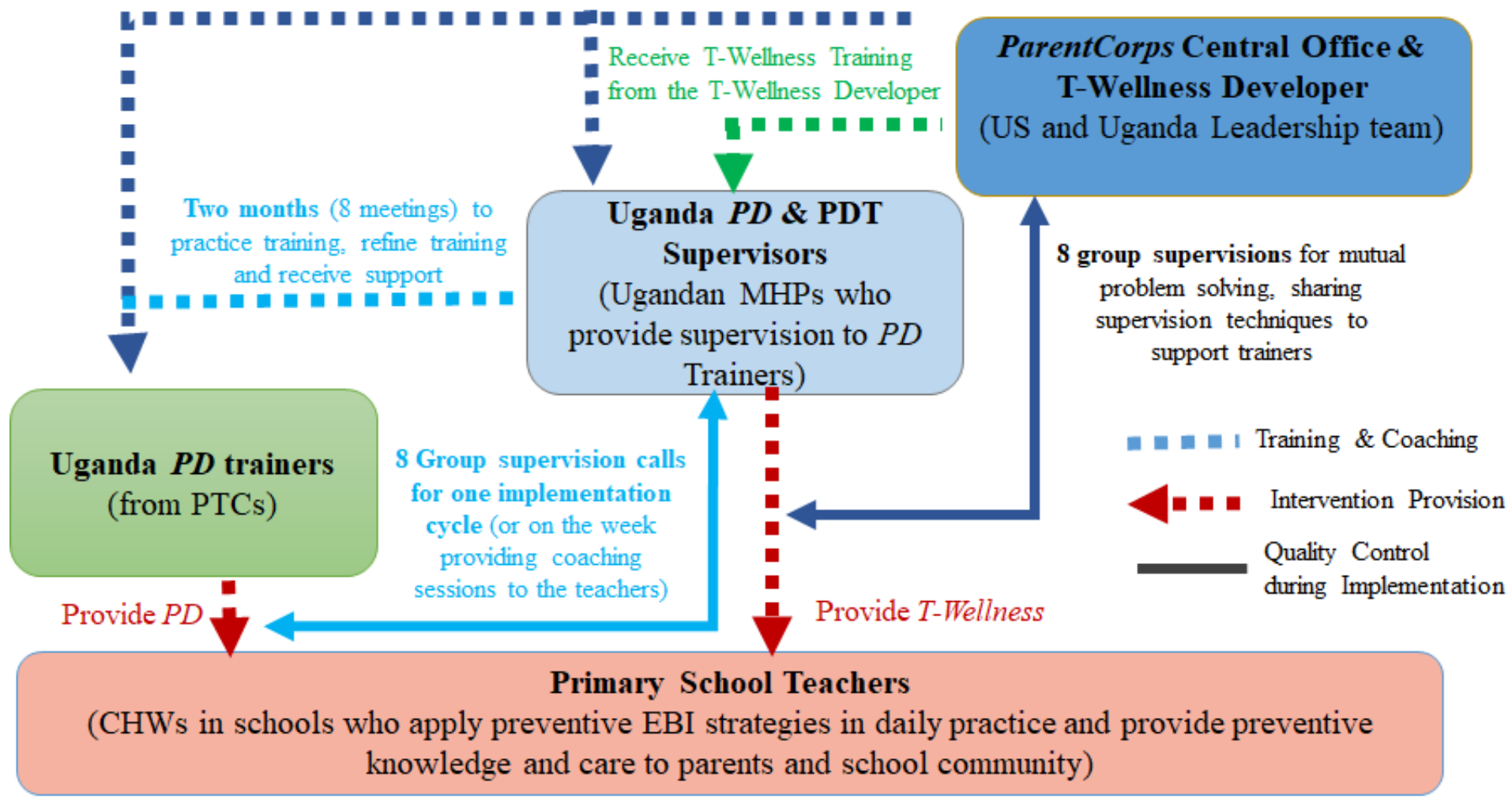

Figure 3

Train-the-Trainers and Supervision Model for Scalable EBI Implementation

\section{Supplementary Files}

This is a list of supplementary files associated with this preprint. Click to download.

- SupplementalNOAIRBApproval.pdf 